Gerhard Stickel

\title{
Zur Förderung von DaF aus der Sicht nationaler Sprachinstitute, speziell des Instituts für Deutsche Sprache (IDS)
}

\section{Warum und wozu fördern?}

Aus der Sicht der deutschsprachigen Länder und Regionen gilt es als nahezu selbstverständlich, dass die deutsche Sprache nicht nur als Erst- und Zweitsprache vermittelt und gefördert wird, sondern auch als Fremdsprache (DaF). Hierzu ein paar Überlegungen, bevor ich auf das mir gestellte Thema näher eingehe. Es liegt dazu nahe, zwischen der Vermittlung und der Förderung einer Sprache zu unterscheiden. Vermittelt wird eine Sprache durch alle Arten von Lehren und Lernen, die dem Erwerb von Kenntnissen der betreffenden Sprache dienen. Gefördert wird eine Sprache auf jede Weise, welche die Vermittlung der Sprache und ihren konkreten Gebrauch ermöglicht und unterstützt. Die Vermittlung selbst ist insoweit unmittelbare Sprachförderung, als sie auf den konkreten Gebrauch der Sprache abzielt und sie damit fördert. Warum sollte Deutsch als Fremdsprache vermittelt und gefördert werden?

Liegt es im Interesse der deutschsprachigen Menschen in Deutschland, Österreich, der Schweiz, der Südtiroler, Ostbelgier und Luxemburger, vielleicht auch der deutschsprachigen Minderheiten in Polen, Ungarn, Namibia, Brasilien und weiteren Ländern, dass auch viele andere Menschen Deutsch lernen und können? Hat es für sie Vorteile? Dies ist als plausibel anzunehmen. Erstsprechern von Deutsch wird durch die Verbreitung von Deutschkenntnissen die Kommunikation mit anderssprachigen Menschen erleichtert. Ein weiterer Vorteil scheint zu sein, dass auf diese Weise auch die Wertschätzung der eigenen Sprache gestärkt wird. Vermutlich wissen aber die allermeisten deutschsprachigen Menschen über die Verbreitung von DaF und seine Förderung nur wenig. Es interessiert sie wohl auch nicht sonderlich, es sei denn in und nach Begegnungen mit Sprechern anderer Sprachen.

Deutlicher ist das Interesse von Politikern, Wissenschaftlern, Künstlern, Geschäftsleuten und Industriellen. Manche Politiker wissen, dass die Verbreitung

Gerhard Stickel, Institut für deutsche Sprache, Mannheim, Deutschland, stickel@ids-mannheim.de 
der eigenen Sprache im Sprachausland ein Indikator der internationalen politischen Stellung des eigenen Landes ist. Sie rechnen damit, dass Menschen, die Deutsch lernen und möglichst auch gebrauchen, positiv gegenüber dem eigenen deutschsprachigen Land eingestellt sind. Wissenschaftler, zumindest in einigen Fächern, sind an der internationalen Kommunikation in der eigenen Sprache interessiert. Künstler, besonders Schriftsteller, möchten auch im Sprachausland wahrgenommen werden, und nicht nur in Ubersetzungen. Kaufleuten und Industriellen erleichtert die eigene Sprache die Kommunikation mit Partnern und Kunden im Ausland.

Wozu wollen anderssprachige Menschen Deutsch lernen? Bringt es ihnen wirtschaftlichen oder kulturellen Gewinn? Begrenzte Umfragen lassen einige Motive als plausibel erkennen. ${ }^{1}$ Deutschkenntnisse sind für Anderssprachige nützlich, die mit deutschsprachigen Menschen zu tun haben oder solche Kontakte wünschen, etwa im Geschäftsleben oder wenn sie in Deutschland oder Österreich arbeiten oder studieren wollen. Es kann auch um Begegnungen mit der deutschen Sprache aus der Ferne gehen: mit Texten, die auf Deutsch verfasst sind, neuer und alter Literatur, Filmen, Fachliteratur, Geschäftskorrespondenz und dergleichen.

Für den schulischen Deutschunterricht im Ausland gibt es zudem ein bildungsbürgerliches Motiv: der oft nur von den Eltern gehegten Wunsch, dass die Kinder und Jugendlichen mit der Kultur der deutschsprachigen Länder vertraut werden. Welche anderssprachigen Menschen lernen also Deutsch und warum? Zum Beispiel sind es:

- Junge Spanier und Griechen, die in deutschsprachigen Ländern Arbeit zu finden hoffen;

- Flüchtlinge aus Kriegsgebieten, die in Deutschland oder Österreich Zuflucht suchen;

- Studenten, die an einer deutschen oder österreichischen Universität studieren (möchten);

- Menschen in den Nachbarstaaten für den grenzüberschreitenden Verkehr;

- Hotel- und Restaurantmitarbeiter, Reiseleiter und Händler in beliebten Urlaubsgebieten;

- Wissenschaftler, die mit deutschsprachigen Kollegen kooperieren (wollen);

- Geschäftsleute und Firmenvertreter, die in Deutschland, Österreich und/oder der Schweiz Geschäfte machen (möchten);

- Bildungsbeflissene Erwachsene und Jugendliche, die sich mit deutscher Literatur im Original befassen möchten;

1 Ausführlich hierzu in Ammon (2015: 987 ff.) 
- Jugendliche, die von klassischer deutscher und österreichischer Musik oder Popmusik fasziniert sind;

- Jugendliche mit deutschsprachigen Vorfahren;

- junge Leute, die sich in eine Deutschsprachige oder einen Deutschsprachigen verliebt haben;

- Linguisten mit einem generellen Interesse an anderen Sprachen, darunter auch Deutsch.

Insgesamt also eine recht bunte Menge von Interessen und Motiven, die zum Teil zusammengehen, denen aber schon wegen ihrer Heterogenität nicht mit einem einfachen Konzept begegnet werden kann. Sie legen es jedoch insgesamt nahe, Deutsch als Fremdsprache zu vermitteln und diese Vermittlung zu fördern. Mehrere deutsche Institutionen sind mit ihren vielfältigen Aktivitäten bemüht, den verschiedenen Gründen und Motiven für das Lehren und Lernen von Deutsch als Fremdsprache zu entsprechen. Sie werden hierfür auf verschiedene Weise gefördert, unter anderem durch staatliche Finanzierung. Die vergleichbaren österreichischen Einrichtungen bleiben im Folgenden unberücksichtigt, weil sie in einem anderen Beitrag behandelt werden. ${ }^{2}$

Auf deutscher Seite ist es vor allem das Goethe-Institut mit seinen Zweigstellen in anderen Ländern und im Inland, das unmittelbar mit der Vermittlung von deutscher Sprache und Kultur und ihrer Förderung in anderssprachigen Ländern befasst ist. Auch die deutschen Auslandsschulen und der Deutsche Akademische Austauschdienst (DAAD) tragen zur Verbreitung und Erhaltung der deutschen Sprache im anderssprachigen Ausland bei. Dies gilt auch für die Zweigstellen der Gesellschaft für deutsche Sprache (GfdS) im Ausland. Hinzu kommen in Deutschland noch die Universitätsinstitute für Deutsch als Fremdsprache und die Germanistikinstitute, die spezielle Studiengänge für DAF anbieten. Von den Studierenden mit entsprechenden Abschlüssen findet ein Teil Aufgaben in der Vermittlung von Deutsch als Fremdsprache.

Hier soll nun eine wissenschaftliche Institution vorgestellt werden, die auf den ersten Blick mit der Förderung oder gar Vermittlung von Deutsch als Fremdsprache nur wenig zu tun hat: das Instituts für Deutsche Sprache (IDS)

2 Vgl. de Cillia in diesem Band. 


\section{Das Institut für Deutsche Sprache und DaF}

Das im Jahr 1964 gegründete Institut für Deutsche Sprache (IDS) in Mannheim ist die zentrale außeruniversitäre Einrichtung in Deutschland zur Erforschung und Dokumentation der deutschen Sprache in ihrem gegenwärtigen Gebrauch und in ihrer neueren Geschichte. Es wird dafür je zur Hälfte von der Bundesregierung und der Ländergemeinschaft finanziert. ${ }^{3}$ Hinzu kommen Projektmittel, unter anderem von der Deutschen Forschungsgemeinschaft (DFG). In den Veröffentlichungen des Auswärtigen Amts zur Förderung von Deutsch als Fremdsprache wird das IDS jedoch nicht erwähnt. ${ }^{4}$ Es hat demnach in der politischen Wahrnehmung mit der Vermittlung und Förderung von DaF nichts zu tun. Tatsächlich hat es damit aber durchaus zu tun, wenngleich nicht als Teil seiner zentralen Aufgaben.

Schon in den Gründerjahren dieses Instituts gab es konkrete Projekte, deren Ergebnisse auch für Anwendungen oder Umsetzungen in der Vermittlung von Deutsch als Fremdsprache gedacht waren. In Zusammenarbeit mit dem GoetheInstitut wurden Elemente für „Grundstrukturen der deutschen Sprache“, auch „Grunddeutsch“ genannt, erarbeitet. Damit war keine vereinfachte Sprache, eine Art Basic German gemeint; es sollten vielmehr analog zum français fondamental die gebrauchshäufigsten grammatischen Strukturen ermittelt und beschrieben werden, die auch für das Sprachenlernen besonders wichtig sind und deshalb in entsprechenden Lehrwerken besonders berücksichtigt werden sollten. Hierbei wurden von Mitarbeitern des IDS linguistische Beschreibungen erarbeitet und von Mitarbeitern des Goethe-Instituts anwendungsbezogene Darstellungen. Nach der Veröffentlichung von über 20 Bänden in der Reihe „Heutiges Deutsch“5 wurde dieses Großvorhaben 1981 beendet, weil die Projektmittel ausliefen. Die meisten der Ergebnisbände sind seitdem aber wiederholt in thematisch verwandten Arbeiten von inländischen und ausländischen Germanisten rezipiert worden, darunter den späteren eigenen grammatischen Forschungen des IDS. Eine unmittelbare Auswirkung auf den Deutschunterricht im anderssprachigen Ausland ist bisher noch nicht untersucht worden, eine mittelbare kann aber als plausibel angenommen werden.

3 Näheres auf der Webseite des Instituts: www.ids-mannheim.de.

4 Auswärtiges Amt (Hrsg.) (1985); Auswärtiges Amt (Hrsg.) (2015); Auswärtiges Amt, Referat 600 (Hrsg.) (2017). Genannt sind dort besonders das Goethe-Institut, der Deutsche Akademische Austauschdienst, die Deutsche Welle und die deutschen Auslandsschulen, nicht aber das IDS.

5 Autoren und Titel sind auf der Webseite des IDS unter http://pub.ids-mannheim.de/abgeschlossen/heutig/ zu finden. 
Dies gilt auch für die Ergebnisse eines weiteren Vorhabens, das in den 70er Jahren hinzukam und das ebenfalls auf Anwendungen im Bereich DaF abzielte: die Erarbeitung kontrastiver Grammatiken, in denen das Deutsche mit je einer anderen Sprache vergleichend beschrieben wurde. Die detaillierten Darstellungen des Deutschen im Vergleich mit jeweils einer anderen Sprache sollten besonders durch den Aufweis der Differenzen und Entsprechungen auf den verschiedenen sprachlichen Strukturebenen als linguistische Grundlagen für die Entwicklung entsprechender Lehrwerke und Unterrichtsverfahren dienen. Erschienen sind seit den 80er Jahren umfangreiche Darstellungen der strukturellen Eigenschaften des Deutschen im Vergleich mit denen des Französischen, Spanischen, Japanischen, Serbokroatischen, Rumänischen und Polnischen. ${ }^{6}$ Die Wahl der Vergleichssprachen war unter anderem bedingt durch die jeweils anderssprachigen Linguisten, die für die Zusammenarbeit gewonnen werden konnten. Zur Vorbereitung und Begleitung der größeren Darstellungen und auch als unmittelbare Folgen wurde eine Vielzahl von kontrastierenden Monographien zu speziellen grammatischen Phänomenen erarbeitet, und zwar zumeist von ausländischen Germanisten. ${ }^{7}$

Gegenüber der zentralen Aufgabenstellung des Instituts, nämlich die deutsche Sprache zu erforschen und zu beschreiben, ließen sich auch diese Arbeiten begründen. Beim Vergleich der eigenen Sprache mit einer anderen werden gerade auch die spezifischen Eigenschaften der eigenen Sprache besonders deutlich. Dies ist auch eine Erfahrung von Lehrerinnen und Lehrern, die ihre eigene Sprache an anderssprachige Lerner vermitteln. Hierbei werden auch ihnen die sprachlichen Strukturen, um die es geht, bewusster als bei unreflektiertem Sprachgebrauch in muttersprachlicher Umgebung. Inwieweit die Ergebnisse der anspruchsvollen kontrastiv-linguistischen Projekte unmittelbar für Lehrwerke oder Unterrichtsmethoden im Bereich Deutsch als Fremdsprache genutzt worden sind, ist schwer festzustellen, zumindest nicht in einer Gesamtschau. Die Projekte haben aber zu intensiver Zusammenarbeit deutscher und ausländischer Germanisten geführt. Letztere waren und sind in ihren Heimatländern nicht nur als Forscher, sondern zumeist auch als Sprachlehrer, oft auch als Lehrwerksau-

6 Zemb (1978/1984); Cartagena \& Gauger (1989); Kaneko \& Stickel (1983/84/87); Engel \& Mrazović (1986); Engel et al. (1993) und Engel et al. (1999).

7 Die meisten dieser Arbeiten (zus. 28) sind in der Reihe „Deutsch im Kontrast“ erschienen; siehe: http://pub.ids-mannheim.de/abgeschlossen/dkontrast/ Hinzu kommt eine Vielzahl von kontrastiv-linguistischen Arbeiten, die von Germanisten im anderssprachigen Ausland geschrieben worden sind, aber vom IDS nicht umfassend bibliographisch erfasst worden sind. 
toren tätig. Eine Anwendung der in gemeinsamer Arbeit mit den deutschen Kollegen ermittelten strukturellen Unterschiede und Gemeinsamkeiten auf Verfahren der konkreten Sprachvermittlung ist deshalb anzunehmen.

Als neueres Projekt, bei dem es um die Darstellung des Deutschen im Vergleich zu mehreren anderen europäischen Sprachen geht, sind die Arbeiten an einer "Grammatik des Deutschen im europäischen Vergleich“ zu nennen. Anstelle eines bilateralen Vergleichs wie in den kontrastiven Grammatiken wurde und wird hierbei das Deutsche mit vier „Kern-Kontrastsprachen“, nämlich Englisch, Französisch, Polnisch und Ungarisch und daneben weiteren europäischen Sprachen verglichen. Als besonders bemerkenswertes Ergebnis ist die umfangreiche „Grammatik des Deutschen im europäischen Vergleich. Das Nominal“ $\mathrm{zu}$ nennen, die 2017 in zwei Bänden erschienen ist. ${ }^{8}$ Auch dieses Werk konnte nur in Zusammenarbeit mit Germanisten aus Ländern mit den verschiedenen Vergleichssprachen geschaffen werden. Neben und vor den genannten beiden Bändern wurden auch von den kooperierenden ,Auslandsgermanisten " vergleichende Studien zu einzelnen grammatischen Teilbereichen erarbeitet. Das Vorhaben ist insgesamt auf eine längerfristige Zusammenarbeit von Linguisten des IDS mit Kolleginnen und Kollegen in mehreren anderen Ländern angelegt. Für die Weiterarbeit konnte vor kurzem eine Kooperation mit der Universität Szeged vereinbart werden für das Teilprojekt „Deutsch-ungarischer Sprachvergleich: korpustechnologisch, funkional-semantisch und sprachdidaktisch (DeutUng)“. Die theoretischen Grundlagen und methodischen Verfahren des Gesamtvorhabens und seiner Teilprojekte können hier nicht gewürdigt werden. Es soll hier nur als besonders markantes Beispiel für die vielen größeren und kleineren wissenschaftlichen Unternehmungen des IDS genannt werden, bei denen es nicht nur um die ,eigene' Sprache geht, diese vielmehr im Kontext anderer europäischer Sprachen untersucht und beschrieben wird, wobei die Eigenschaften des Deutschen sowohl deutschen als auch anderssprachlichen Linguisten besonders vertraut gemacht wurden und werden. Dies nützt den ausländischen Beteiligten wie auch den Adressaten der Ergebnisse, zu deren Aufgaben die Vermittlung des Deutschen als Fremdsprache gehört.

Unter den derzeit laufenden Projekten sei noch ein Vorhaben genannt, bei dem die Nutzung lexikalischer Hilfsmittel im Unterricht von Deutsch als Fremdsprache und speziell in der konkreten Textproduktion untersucht wird. Begleitet wird dieses Projekt von einer europaweiten Erhebung zur Wörterbuchbenutzung und, Wörterbuchkultur. An beiden Projekten sind Partnerinnen und Partner aus

8 Gunkel, Murelli, Schlotthauer, Wiese \& Zifonun (2017). 
dem In- und Ausland beteiligt. ${ }^{9}$ Auch diese wissenschaftliche Unternehmung trägt also zur Förderung von $\mathrm{DaF}$ bei.

Neben den verschiedenen Kooperationen des IDS mit Universitätsinstituten in anderssprachlichen Ländern ist zudem zu erwähnen, dass einzelne wissenschaftliche Mitarbeiterinnen und Mitarbeiter des IDS für kürzere oder längere Zeit vom Institut beurlaubt wurden und werden, um als Dozenten oder Lektoren für $\mathrm{DaF}$ an ausländischen Universitäten tätig zu werden. Den zentralen Aufgaben des IDS dient ihre Erfahrung, die eigene Sprache für eine Zeitlang auch aus der Fremdperspektive wahrzunehmen, ein Vorteil, den sie nach ihrer Rückkehr für ihre Forschungen zum Deutschen nutzen können.

Einen mittelbaren Nutzen für den Bereich DaF hat das IDS zudem als zentraler Bezugsort für Germanisten und Deutschlehrer aus anderssprachlichen Ländern. Die Mehrzahl der Mitglieder des Internationalen Wissenschaftlichen Rats des Instituts sind Hochschulgermanisten aus anderen Ländern. Sie gewinnen aus dem Kontakt mit dem IDS und den Verbindungen untereinander Impulse für ihre Forschungen und die Ausbildung von Deutschlehrern in ihren Ländern. Mit ihrer Hilfe wurden in den Jahren 2004, 2006 und 2008 jeweils Dokumentationen über die Germanistik und den Deutschunterricht in mehreren Ländern erarbeitet und publiziert. ${ }^{10} \mathrm{Zu}$ hoffen ist, dass diese Dokumentationen fortgesetzt werden können. Das IDS wird außerdem gesucht und besucht von meist jüngeren Auslandsgermanisten als Forschungszentrum für längere oder kürzere Forschungsaufenthalte. Allein im Jahr 2016 waren 145 ausländische Gastwissenschaftlerinnen und -wissenschaftler im IDS tätig. Auch sie gewinnen hierbei nicht nur Anregungen für ihre speziellen Forschungsthemen, sondern auch für die praktische Vermittlung von DaF.

Stand und Verbreitung von Deutsch als Fremdsprache gehören zu den wiederkehrenden Themen des Deutschen Sprachrats, in dem das Institut für Deutsche Sprache mit den beiden wichtigen Einrichtungen für die Vermittlung von DaF, dem Goethe-Institut und dem Deutschen Akademischen Austauschdienst sowie der Gesellschaft für deutsche Sprache zusammenarbeitet ${ }^{11}$.

An alle dem zeigt sich, dass das Institut für Deutsche Sprache an der unmittelbaren Vermittlung von Deutsch als Fremdsprache zwar kaum beteiligt ist; es

$9 \mathrm{Zu}$ Kurzbeschreibungen der Teilprojekte siehe http://www1.ids-mannheim.de/lexik/empirische-methoden/kooperationen.html.

10 Siehe das Publikationsverzeichnis des IDS unter http://pub.ids-mannheim.de/laufend/sonstige/titel1.html.

11 Näheres unter: www.deutscher-sprachrat.de. 
trägt aber in vielfältiger, durchweg mittelbarer Weise durch seine Projekte, Kooperationen und Kontakte zur Vermittlung und dem Gebrauch von Deutsch als Fremdsprache bei. Dies zudem aus wissenschaftlichem ,Eigennutz; denn es profitiert damit auch für seine zentralen Aufgaben, die Erforschung und Dokumentation der eigenen Sprache.

Kurz erwähnt werden soll noch eine internationale Organisation, der das IDS angehört, die ebenfalls einen mittelbaren, wenn auch weniger deutlichen Nutzen für die Wahrnehmung des Deutschen aus der sprachlichen Außenperspektive, also des Deutschen als Fremdsprache hat.

\section{EFNIL}

Seit 2003 besteht eine europäische Vereinigung nationaler Sprachinstitutionen: EFNIL (European Federation of National Institutions for Language). ${ }^{12}$ Diese Organisation vereint die Sprachakademien und zentralen Sprachinstitute der Staaten der Europäischen Union - darunter das IDS - und weiterer europäischer Länder. Die einzelnen Mitgliedseinrichtungen haben die Aufgabe, sich wissenschaftlich beschreibend und/oder sprachplanerisch um die offiziellen Sprachen ihrer Länder zu kümmern. Sie sind im Grunde genommen Sprachegoisten; denn sie sind in ihrer Aufgabenstellung auf die eigenen Sprachen fokussiert. So ist zum Beispiel die Accademia della Crusca in Florenz seit Jahrhunderten nur mit dem Italienischen befasst, der Dansk Sprognævn (Dänische Sprachrat) nur mit dem Dänischen und das Institut für Deutsche Sprache - wie schon erwähnt - hauptsächlich mit dem Deutschen.

Sonderfälle unter den Mitgliedsinstitutionen von EFNIL sind die Nederlandse Taalunie (Niederländische Sprachunion), die sich mit der niederländischen Sprache sowohl in den Niederlanden als auch in Flandern befasst. Dieser binationalen Einrichtung geht es aber ebenfalls nur um die eigene Sprache in den beiden politischen Gebieten. Zum Aufgabengebiet des Kotimaisten kielten keskus/Institutet för de inhemska språken (Institut für die Sprachen Finnlands) in Helsinki gehören neben den beiden offiziellen Sprachen Finnisch und Schwedisch auch die einheimischen Minderheitssprachen in Finnland. Hierbei sind es also mehrere eigene Sprachen. Das gilt auch für das Institut für Mehrsprachigkeit in Fribourg, das das Miteinander der vier offiziellen Sprachen der Schweiz untersucht

12 Näheres zu den Zielen, Aktivitäten und Mitgliedsinstitutionen unter: www.efnil.org. 
und beschreibt. Weitere Sonderfälle sind der British Council und das portugiesische Instituto Camões, die nur mittelbar mit Sprachbeschreibung und Sprachplanung befasst sind. Ihre Aufgabe ist ähnlich wie die des deutschen Goethe-Instituts die Verbreitung der eigenen Sprache im Ausland. Sie gehören zu EFNIL anstelle einer Sprachakademie oder einer anderen zentralen Einrichtung für die offiziellen Landessprachen der beiden Länder. Auch ihnen geht es aber gezielt um die eigenen Sprachen.

Wenngleich alle Mitgliedseinrichtungen von EFNIL, einschließlich der genannten Sonderfälle, ausschließlich oder hauptsächlich mit den eigenen Sprachen befasst sind, eint sie das Bemühen, die eigenen Sprachen im Kontext der anderen europäischen Sprachen zu erforschen und zu fördern. Sie gehen von der Uberzeugung aus, dass die eigenen Sprachen nur in diesem Kontext eine gute Zukunft haben, also als Teile der europäischen Sprachenvielfalt. Dass diese Sprachenvielfalt eine wesentliche Bedingung für den kulturellen Reichtum und die soziale Vielfalt Europas ist, gehört seit langem zum gemeinsamen Europadiskurs.

Mit Ausnahme des erwähnten Instituto Camões und des British Council sind die EFNIL-Institute nicht aktiv mit der Verbreitung der eigenen Sprachen als Fremdsprachen im anderssprachigen Ausland befasst. Es ist zumindest nicht ihre Hauptaufgabe. Ihre laufenden Arbeiten und Projekte richten sich in erster Linie auf die eigenen Sprachen im Sprachinland. Sie erforschen und dokumentieren die eigenen Sprachen, und - je nach Aufgabenstellung - planen und pflegen sie diese Sprachen auch etwa durch die Beratung von Schulbehörden oder die Erarbeitung von Materialien für den Sprachunterricht. Das Lehren, Lernen und der Gebrauch dieser Sprachen als Fremdsprachen ist dabei allenfalls ein Nebenaspekt.

In der konkreten Zusammenarbeit innerhalb von EFNIL nehmen die einzelnen Institute jedoch in besonderer Weise Rücksicht auf die Sprachen der anderen Institute, d.h. die jeweils anderen Fremdsprachen. Die vorherrschende Arbeitssprache bei Tagungen und Sitzungen von Gremien und Projektgruppe ist zwar Englisch, weil dies die einzige Sprache ist, in der sich alle Vertreter aus den verschiedenen Mitgliedsinstituten wenigstens einigermaßen verständigen können. Für Vorträge auf Tagungen und Publikationen ist aber Mehrsprachigkeit vorgesehen. Auf den Jahrestagungen gibt es Dolmetscherdienste für wenigstens drei, oft auch vier Sprachen, wobei freilich meist die drei Arbeitssprachen der EU-Kommission Englisch, Französisch und Deutsch bevorzugt werden, dies unter anderem mit dem Argument, dass diese drei Sprachen am ehesten auch von Sprechern anderer Sprachen verstanden werden. Für Deutsch gilt zudem, dass es offizielle Sprache oder eine von mehreren offiziellen Sprachen in fünf der Staaten ist, de- 
ren Sprachinstitutionen Mitglieder von EFNIL sind: Belgien, Deutschland, Luxemburg, Österreich und Schweiz. Resolutionen und Empfehlungen von EFNIL zur Europäischen Sprachenpolitik werden in den offiziellen Sprachen aller oder der meisten Mitgliedsinstitute veröffentlicht (http://efnil.org/documents). In Buchpublikationen zu den Jahrestagungen werden die Resümees oder Abstracts zu den Aufsatzbeiträgen auch in den Muttersprachen der einzelnen Autoren gefasst. ${ }^{13}$

Zur Förderung der deutschen Sprache als Fremdsprache trägt EFNIL mittelbar bei, als es den Respekt, vielleicht sogar die Sympathie der Vertreter der anderen europäischen Sprachinstitute für die deutsche Sprache verstärkt. Die Initiative zur Gründung dieser europäischen Organisation ging von Institut für Deutsche Sprache aus, und der Vertreter des IDS ist Vorsitzender von EFNIL seit deren Gründung. Deutsch wird auch in den internationalen Projekten von EFNIL jeweils berücksichtigt. Weil aber Englischkenntnisse unter den Mitgliedern erheblich weiter verbreitet sind als Deutschkenntnisse, ergibt sich ganz praktisch, dass die deutsche Sprache in diesem Kontext zwar eine besondere, aber doch nachrangige Stellung hat.

Das Institut für Deutsche Sprache fördert also auch in Verbindung mit EFNIL die internationale Stellung des Deutschen. Hinzu kommen, wie gezeigt wurde, seine Projekte und internationalen Kooperationen, darunter auch solchen mit anderen Mitgliedsinstituten von EFNIL, wodurch die Verbreitung des Deutschen als Fremdsprache gefördert wird. Das IDS sollte deshalb in Beschreibung, Diskussion und Planung der Förderung von Deutsch als Fremdsprache stärker als bisher berücksichtigt werden.

\section{Literaturverzeichnis}

Ammon, Ulrich (2015): Die Stellung der deutschen Sprache in der Welt. Berlin u.a.: de Gruyter. Auswärtiges Amt (Hrsg.) (1985): Bericht der Bundesregierung Oktober - Die Stellung der deutschen Sprache in der Welt. Bonn.

Auswärtiges Amt (Hrsg.) (2015): Deutsch als Fremdsprache weltweit. Datenerhebung 2015. Berlin.

Auswärtiges Amt (Hrsg.) (2017): Die Förderung von Deutsch als Fremdsprache im Ausland. Wer macht was im Rahmen der AKBP? Berlin.

Cartagena, Nelson \& Hans-Martin Gauger (1989): Vergleichende Grammatik Spanisch-Deutsch. 2 Bände, Mannheim u.a.: Bibliographisches Institut.

13 Siehe als Beispiele die zuletzt erschienen Bände Nuolijärvi \& Stickel (2016) und Dąbrowska, Pisarek \& Stickel (2017). 
Dąbrowska, Anna, Walery Pisarek \& Gerhard Stickel (Hrsg.) (2016): Stereotypes and linguistic prejudices in Europe. Budapest: Research Institute for Linguistics. Hungarian Academy of Sciences.

Gunkel, Lutz, Adriano Murelli, Susan Schlotthauer, Bernd Wiese \& Gisela Zifonun (2017): Grammatik des Deutschen im europäischen Vergleich. Das Nominal. Unter Mitarbeit von Christine Günther und Ursula Hoberg. Berlin, Boston: de Gruyter.

Engel, Ulrich \& Pavica Mrazović (Hrsg.) (1986): Kontrastive Grammatik: Deutsch-Serbokroatisch. 2 Bde., München: Otto Sagner.

Engel, Ulrich et al. (1993): Kontrastive Grammatik deutsch-rumänisch. 2 Bde., Heidelberg: Julius Groos.

Engel, Ulrich et al. (1999): Kontrastive Grammatik deutsch-polnisch. 2 Bde., Heidelberg: Julius Groos.

Kaneko, Tohru \& Gerhard Stickel (Hrsg.) (1983/84/87): Deutsch und Japanisch im Kontrast. Heidelberg: Julius Groos.

Nuolijärvi, Pirkko \& Gerhard Stickel (Hrsg.) (2016): Language use in public administration. Budapest: Research Institute for Linguistics, Hungarian Academy of Sciences.

Zemb, Jean-Marie (1978/1984): Vergleichende Grammatik Französisch-Deutsch. 2 Bände, Mannheim u.a.: Bibliographisches Institut. 